\title{
Hard fast X-ray transients as possible counterparts of unidentified MeV/TeV sources
}

\author{
V. Sguera*t \\ INAF/IASF Bologna, via Piero Gobetti 101, 40129 Bologna, Italy \\ E-mail: sguera@iasfbo.inaf.it
}

\begin{abstract}
The two still unidentified MeV sources EGR J1122-5946 and AGL J2022+3622 are here tentatively associated with soft gamma-ray candidate counterparts detected through INTEGRAL/IBIS observations. On the basis of spatial proximity and/or similar transient behaviour, we propose the supergiant fast X-ray transient (SFXT) IGR J11215-5952 and the candidate SFXT IGR J20188+3647 as possible candidate counterparts of EGR J1122-5946 and AGL J2022+3622, respectively. Our findings possibly suggest that hard fast X-ray transients could represent a new class of galactic transient $\mathrm{MeV} / \mathrm{TeV}$ emitters. Additional evidence for the existence of such new class is also provided by very recent AGILE and GLAST discoveries on the galactic plane of several unidentified transient $\mathrm{MeV}$ sources lasting only a few days
\end{abstract}

7th INTEGRAL Workshop

September 8-11 2008

Copenhagen, Denmark

\footnotetext{
* Speaker.

${ }^{\dagger}$ I am very grateful to L. Bassani, A. Bazzano, A.J. Bird and G.E. Romero for their collaboration. This research made use of data obtained from the HEASARC database.
} 


\section{Introduction}

The field of high energy astronomy is relatively new and young. Breakthrough results have been obtained in the last twenty years thanks to gamma-ray satellites carrying instruments such as CGRO/EGRET, INTEGRAL/IBIS and Swift/BAT whose survey capabilities unveiled the extreme richness of objects in the gamma-ray sky (Hartman et al. 1999, Bird et al. 2007, Casandjian \& Grenier 2008). Recently, ground-based TeV astronomy has also shown rapid progress with important results reported by the third generation of imaging atmospheric Cherenkov telescopes such as HESS, MAGIC, VERITAS and CANGAROO. A rapidly growing list of $\sim 75 \mathrm{TeV}$ sources have been detected, $\sim 35 \%$ of which are still unidentified (Ribo 2008)

Among the different types of sources shining in the high energy sky, High Mass X-ray Binaries (HMXBs) rapidly became the subject of topical and major interest in high energy astronomy. During the last few years, four HMXBs have been detected at TeV energies, namely Cygnus X-1 (Albert et al. 2007), LS 5039 (Aharonian et al. 2005a), LS I+61 303 (Albert et al. 2006) and PSR B1259-63 (Aharonian et al. 2005b); their TeV emission provides evidence that particles can be efficiently accelerated to very high energies. Furthermore, in the last few years the IBIS instrument (Ubertini et al. 2003) on board the INTEGRAL satellite (Winkler et al. 2003) has revolutionised our classical view of HMXBs previously built over $\sim 40$ years of X-ray observations. Among the others, IBIS has discovered many supergiant HMXBs (SGXBs) with X-ray characteristics never seen before; they represent a new class of X-ray binaries which have been named as Supergiant Fast X-ray Transients, SFXTs (Sguera et al. 2005,2006, Negueruela et al. 2006). SFXTs spend most of the time in a low level of X-ray activity characterized by luminosities in the range $10^{32}-10^{34} \mathrm{erg}$ $\mathrm{s}^{-1}$, well below the persistent state of classical SGXBs $\left(\sim 10^{36} \mathrm{erg} \mathrm{s}^{-1}\right)$. Only occasionally they do display fast X-ray flares, lasting typically a few hours, with a dinamical range of $\sim 10^{3}-10^{4}$. Two main models have been proposed to explain their peculiar fast X-ray flaring behaviour, they invoke spherically simmetric clumpy wind from the supergiant companion (Negueruela et al. 2008, Walter \& Zurita 2007) and anisotropic wind (Sidoli et al. 2007), respectively.

A common characteristic of the IBIS and EGRET catalogs (Hartmann et al. 1997, Bird et al. 2007, Casandjian \& Grenier 2008) is that a considerable fraction of their high energy sources is still unidentified, with no counterpart at other wavelengths. This indicates the need for continuing their studies with current missions such as INTEGRAL, GLAST, AGILE, in order to find counterparts capable to produce this high energy radiation. To this aim, very recently Sguera et al. (2008 submitted) proposed the SFXT AX J1841.0-0536 as a fast transient MeV/TeV emitter, based on spatial proximity and similar X-ray/soft $\gamma$-ray behavior with the unidentified sources 3EG J1837-0423 and HESS J1841-055. Such association is also supported from an energetic standpoint by a theoretical scenario where AX J1841.0-0536 is a low magnetized pulsar which, due to accretion of a massive clump from its supergiant companion donor, undergoes sporadic changes to a transient state where a magnetic tower can produce transient jets and as a consequence high energy emission (Sguera et al. 2008 submitted). Based on their findings, the authors proposed the SFXT AX J1841.0-0536 as the prototype of a new class of galactic fast transient MeV/TeV emitters. Additional evidences for the existence of such new class is also provided by very recent AGILE and GLAST discoveries on the galactic plane of several unidentified transient MeV sources lasting only a few days (Chen et al. 2007, Pittori et al. 2008, Longo et al. 2008, Cheung et 


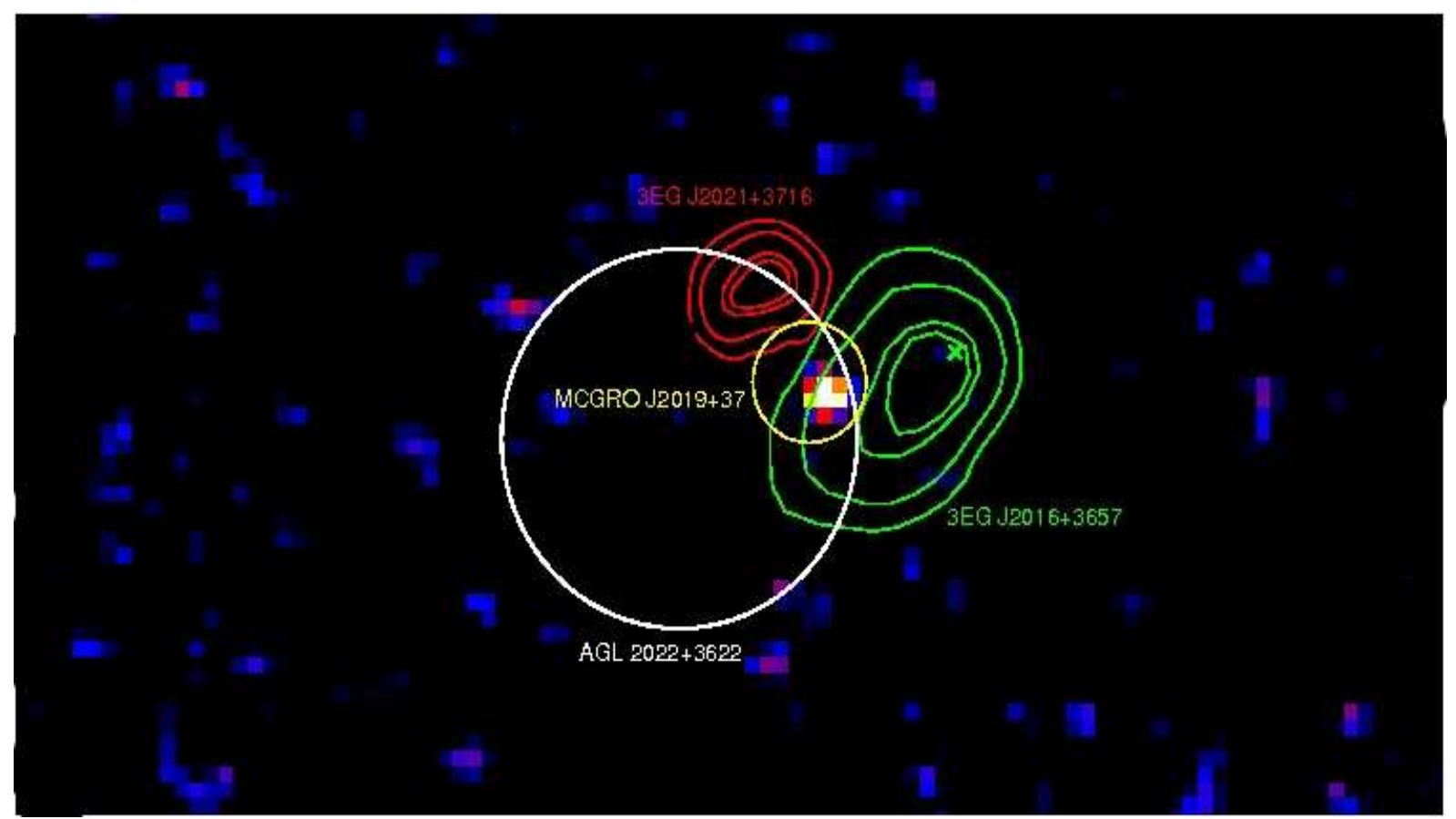

Figure 1: IBIS $17-30 \mathrm{keV}$ significance image ( $\sim 1$ hour exposure) of the fast hard $\mathrm{X}$-ray transient IGR J20188+3647 ( $\sim 7 \sigma$ detection) with superimposed: AGL J2022+3622 error circle in white, MGRO J2019+37 error circle in yellow, 3EG J2016+3657 probability contours in green (from 50\% to 99\%) and 3EG J2021+3716 probability contours in red (from 50\% to 99\%).

al. 2008).

Here we propose two other fast X-ray transients, IGR J11215-5952 and IGR J20188+3647, as best candidate counterparts of two unidentified MeV sources, EGR J1122-5946 and AGL $\mathrm{J} 2022+3622$ respectively. Our proposed associations are mainly based on spatial correlation and/or similar temporal behaviour.

\section{AGL J2022+3622}

The transient MeV source AGL J2022+3622 (E>100 MeV) was discovered in the Cygnus region by AGILE on 23 November 2007 (Chen et al. 2007). Its positional error circle is centered at galactic coordinates $1=75^{\circ} .0, b=-0^{\circ} .4$ with an error radius of $\sim 1^{\circ}$. AGL J2022 +3622 was characterized by a strongly variable gamma-ray emission lasting only one day.

\subsection{IBIS observations of AGL $2022+3622$}

Unfortunately, no simultaneous IBIS observations of the AGILE source's discovery are available. The region of the sky including AGL J2022+3622 was observed by IBIS $\sim 9$ days after the AGILE discovery for $1 \sim$ Ms but no hard X-ray source was detected inside the AGILE error circle, providing a $2 \sigma$ upper limit of $\sim 0.4 \mathrm{mCrab}(20-40 \mathrm{keV})$. To date, the region of the sky including AGL J2022+3622 has been observed by IBIS for a total of $\sim 2$ Ms. Only in one occasion, on 13 July 2004, IBIS detected a newly discovered fast hard X-ray transient source that is located 


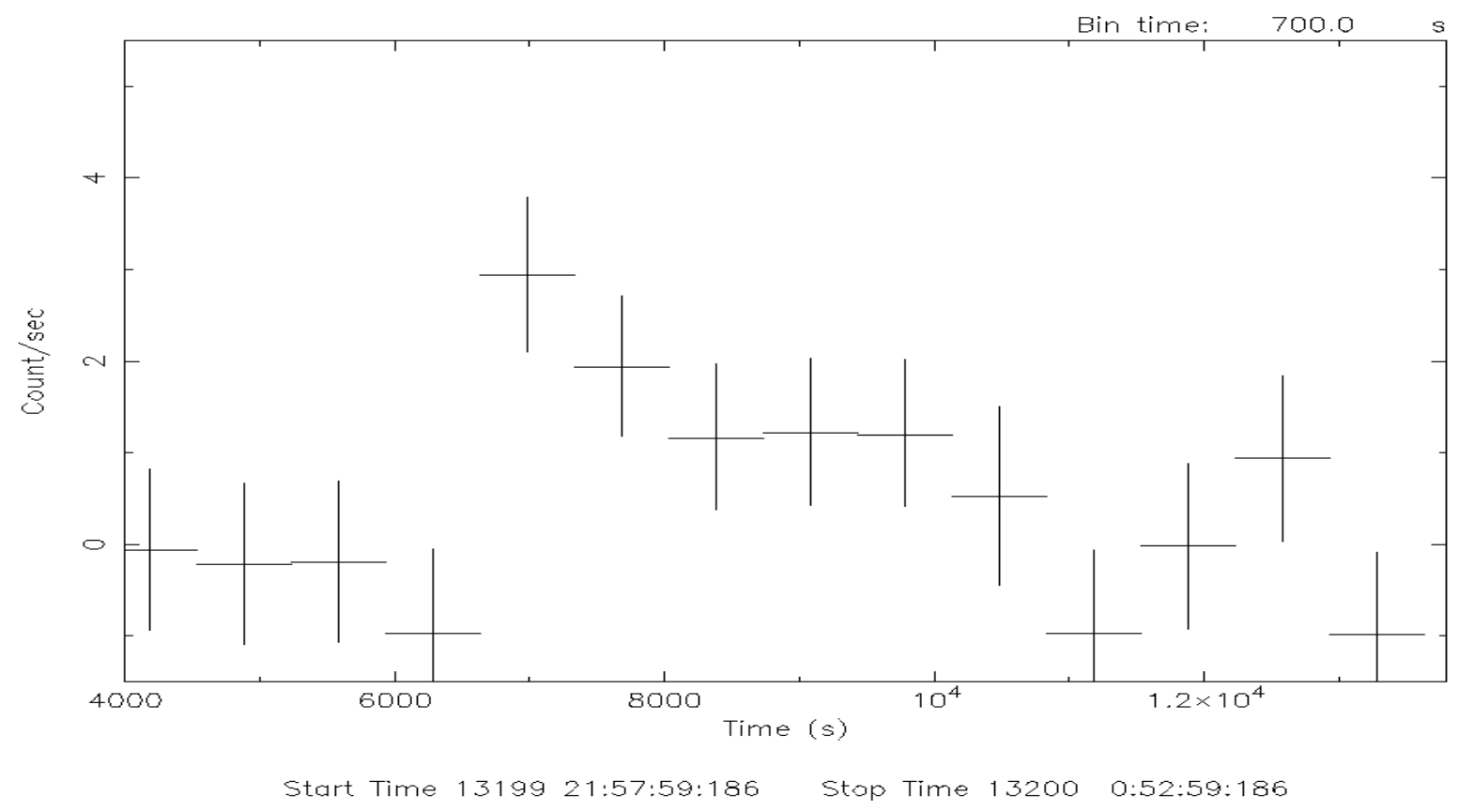

Figure 2: ISGRI 17-30 keV light curve of the fast hard X-ray transient IGR J20188+3647 pertaining to its discovery by IBIS.

inside the AGILE error circle: IGR J20188+3647 (Sguera et al. 2006b). This source is clearly detected at $\sim 7 \sigma$ level $(17-30 \mathrm{keV})$ in the IBIS significance image shown in fig. 1 ( $\sim 1$ hour exposure time) at coordinates $\mathrm{RA}=201848, \mathrm{Dec}=3647$ 31.2, error radius $=3.4$ arcminutes $(90 \%$ confidence). The average flux is equal to $\sim 33 \mathrm{mCrab}$ or $2.1 \times 10^{-10} \mathrm{erg} \mathrm{cm}^{-2} \mathrm{~s}^{-1}(17-30 \mathrm{keV})$. We note that the poorly known and unidentified EXOSAT source EXMS B2016+366 (Reynolds et al. 1999) is located inside the error circle of IGR J20188+3647. Furthermore, IGR J20188+3647 is located inside the error box of the poorly known and unidentified HEAO A-1 source 1H2018+366 (Wood K.S. et al. 1984). It could be that the three hard X-ray sources are the same object. Fig. 1 clearly shows that the Agile source AGL J2022+3622 and IGR J20188+3647 are spatially correlated. IGR J20188+3647 is the only hard X-ray source $(\mathrm{E}>10 \mathrm{keV})$ located inside the AGILE positional uncertainty.

Although the statistics are not good enough to perform a detailed spectral analysis, we were able to extract an ISGRI spectrum of IGR J20188+3647 (17-30 keV) from the unique Science Window $(\mathrm{ScW})$ during which it was detected $\left(\sim 1\right.$ hour exposure time). A power law $\left(\chi_{v}^{2}=1.6\right.$, d.o.f 3), or alternatively a thermal bremsstrahlung $\left(\chi_{v}^{2}=1.7\right.$, d.o.f 3$)$, gave reasonable fits although it was not possible to fully constrain the spectral parameters ( $\Gamma \sim 2.4$ and $\mathrm{kT} \sim 14 \mathrm{keV}$, respectively). Fig. 2 shows the ISGRI light curve (17-30 keV) of IGR J20188+3647, it is evident the transient behaviour with a fast rise $(\sim 10$ minutes) followed by a slower decay ( $\sim 50$ minutes). This kind of fast X-ray flaring activity and spectral parameters strongly resemble those of SFXTs and we can consider it a candidate SFXT.

It is worth noting that IGR J20188+3647 and AGL J2022+3622 are located in a region of the sky rich of other high energy sources, as can be clearly seen in fig.1. Specifically, we highlight the presence of the following gamma-ray sources: 
- MGRO J2019+37 (smaller and yellow circle in fig. 1). It is an unidentified and highly extended $\mathrm{TeV}$ source discovered by the water Cherenkov detector Milagro (Abdo et al. 2007). Although fig. 1 clearly shows that MGRO J2019+37 is spatially correlated with IGR J20188+3647, the extended and diffuse nature of the $\mathrm{TeV}$ source seems to exclude a physical association.

- 3EG J2016+3657 (green probability contours at 50\%, 68\%, 95\% and 99\%). It has been optically identified by Halpern et al. (2001) with the blazar B2013+370 indicated by the green $x$ point in fig. 1, so we can rule out a physical association between 3EG J2016+3657 and IGR J20188+3647.

- 3EG J2021+3716 (red probability contours at 50\%, 68\%, 95\% and 99\%). Very recently, AGILE observations $(E>100 \mathrm{MeV})$ identified its counterpart with the pulsar PSR J2021+3651 (Halpern et al. 2008) that does not show variability at gamma-rays.

It is worth pointing out that the above EGRET sources are from the third EGRET catalog (Hartman et al. 1997). Comparing with the very recent and revised EGRET catalog by Casandjian \& Grenier (2008), we note that 3EG J2016+3657 is not listed anymore while 3EG J2021+3716 is still listed with coordinates similar to those from the third EGRET catalog.

In the light of all the findings reported above, we propose the candidate SFXT IGR J20188+3647 as one possible candidate counterpart of the fast transient gamma-ray source AGL J2022+3622, to date. Further and deep studies of the entire region in X-rays (i.e. XMM, Chandra and Swift/XRT), soft gamma-rays (i.e. INTEGRAL), $\mathrm{MeV}$ and GeV (i.e. AGILE and GLAST) are strongly needed in order to confirm or reject our proposed identification.

\section{EGR J1122-5946}

Recently, the whole EGRET dataset $(\mathrm{E}>100 \mathrm{MeV})$ has been reanalyzed by using a new and much improved galactic interstellar emission model based on very recent dark gas, $\mathrm{CO}, \mathrm{HI}$, and interstellar radiation field data. The results have been reported by Casandjian \& Grenier (2008) in a revised EGRET catalog which lists 188 sources compared to the 271 entries of the previous third EGRET catalog. About 107 former EGRET sources have not been confirmed because of structures in the interstellar background, the majority of them were unidentified sources associated with the local clouds of the Gould belt. It is worth pointing out that the revised catalog by Casandjian \& Grenier (2008) also lists 30 new gamma-ray sources not previously reported in the third EGRET catalog. Among them, we focussed our attention on the newly discovered and unidentified gammaray source EGR J1122-5946. It is located at $\mathrm{RA}=170^{\circ} .55$ and $\mathrm{Dec}=-59^{\circ} .77$ with an error circle radius of $0^{\circ} .31$. Unfortunately, it is still unknown if EGR J1122-5946 is a persistent or a transient gamma-ray object since no such information is provided in the revised catalog. However from the reported fluxes $(E>100 \mathrm{MeV})$ in three different EGRET viewing period lasting $\sim$ one year

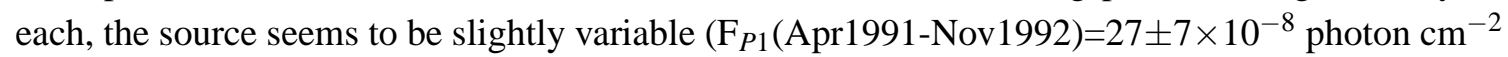
$\mathrm{s}^{-1}, \mathrm{~F}_{P 3}\left(\right.$ Aug1993-Oct1994) $=9 \pm 8 \times 10^{-8}$ photon $\mathrm{cm}^{-2} \mathrm{~s}^{-1}, \mathrm{~F}_{P 4}\left(\right.$ Oct1994-Oct1995) $=53 \pm 15 \times 10^{-8}$ photon $\mathrm{cm}^{-2} \mathrm{~s}^{-1}$ ). 


\subsection{IBIS observations of EGR J1122-5946}

The region of the sky including EGR J1122-5946 has been extensively covered by IBIS observations and we exploited them with the aim of finding its best candidate counterpart. Fig. 3 shows the 20-100 keV IBIS significance mosaic ( 2.5 Ms exposure) superimposed on 95\% EGRET error circle. We note that no persistent soft gamma-ray source has been detected inside of it despite the deep IBIS observation. As next step, we took into account the possibility that a fast X-ray transient source, active on short timescale (i.e. few hours), could be located inside the error circle of EGR J1122-5946 and it was not detected in the deep IBIS mosaic because the integration on long exposure time degrades the signal to noise to very low values. Bearing such possibility in mind, we performed an analysis at ScW level $(\sim 2 \mathrm{ks})$ of the entire IBIS ScW dataset $(\sim 2.5 \mathrm{Ms})$. By doing so we unveiled the presence of the SFXT IGR J11215-5952 inside the EGR J1122-5946 error circle. This can be clearly seen in fig. 4 which shows the 20-100 keV IBIS significance mosaic taking into account only the ScWs during which IGR J11215-5952 was active ( $\sim 45 \mathrm{ks}$ exposure time). No other catalogued hard X-ray sources $(E>10 \mathrm{keV})$ are located inside the EGR J1122-5946 error circle, according with all the available catalogs from the HEASARC database.

IGR J11215-5952 is a HMXB binary system hosting a $\sim 187$ seconds neutron star as compact object and a massive supergiant star as companion donor. It is characterized by a fast X-ray transient behaviour (Sidoli et al. 2006,2007, Romano et al. 2007) and classified as SFXT. The orbit of IGR J11215-5952 is highly eccentric and the neutron star spends most of the time far away from the companion donor; the typical quiescent luminosity of the system is $\mathrm{L}_{x} \sim 10^{33} \mathrm{erg}$ $\mathrm{s}^{-1}$. Accretion takes place only at periastron passage (every $\sim 165$ days), when the compact object approaches the supergiant star; the brightest part of the outburst activity consists of fast X-ray flares ( $\sim$ hours) with a typical X-ray luminosity of $10^{36} \mathrm{erg} \mathrm{s}^{-1}$.

As we can clearly see in fig. 4, IGR J11215-5952 is spatially correlated with the unidentified EGRET source EGR J1122-5946 and this makes tempting to postulate a physical correlation between the two objects. Unfortunately, informations on the temporal behaviour of EGR J1122-5946 (i.e. persistent or transient nature), which could shed more light on its association with the SFXT, are still unknown and a further study from this point of view is strongly needed. Finally, we took into account the possibility that the spatial association could be simply a chance coincidence. To this aim, we calculated the probability of finding a supergiant HMXB, such as IGR J11215-5952, inside the EGR J1122-5946 error circle $\left(\sim 0^{\circ} .3\right)$ by chance. Given the number of supergiant HMXBs detected by IBIS within the galactic plane (Bird et al. 2007), defined here as a latitude range $\pm 3^{\circ}$, we estimated a probability of $\sim 0.6 \%$, i.e. low enough to claim a physical association between EGR J1122-5946 and IGR J11215-5952.

\section{Discussion and future work}

In the light of the findings previously reported, we propose the SFXT IGR J11215-5952 and the candidate SFXT IGR J20188+3647 as possible candidate counterparts of the unidentified MeV sources EGR J1122-5946 and AGL J2022+3622, respectively. It is worth pointing out that the possible fast MeV flares from IGR J11215-5952 and IGR J20188+3647 might not be a unique and rare case. Another SFXT, AX J1841.0-0536, has also been proposed as a fast transient MeV/TeV emitter based on spatial proximity and similar X-ray/soft $\gamma$-ray behavior with the unidentified sources 


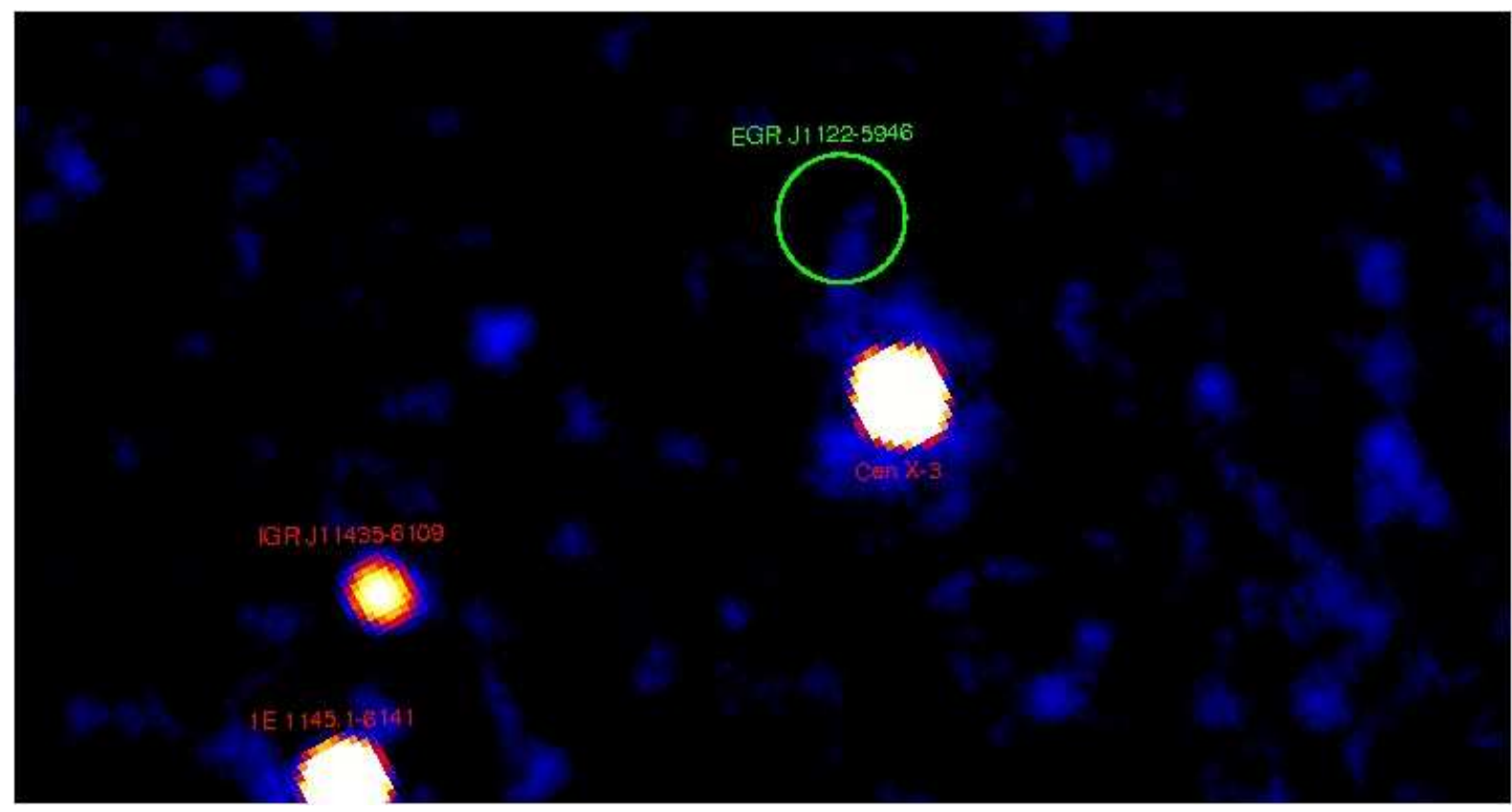

Figure 3: IBIS 20-100 keV significance deep mosaic image ( $\sim 2.5 \mathrm{Ms})$ superimposed on the EGR J1122-5946 error circle. We note that no persistent soft gamma-ray source has been detected inside the EGRET error circle.

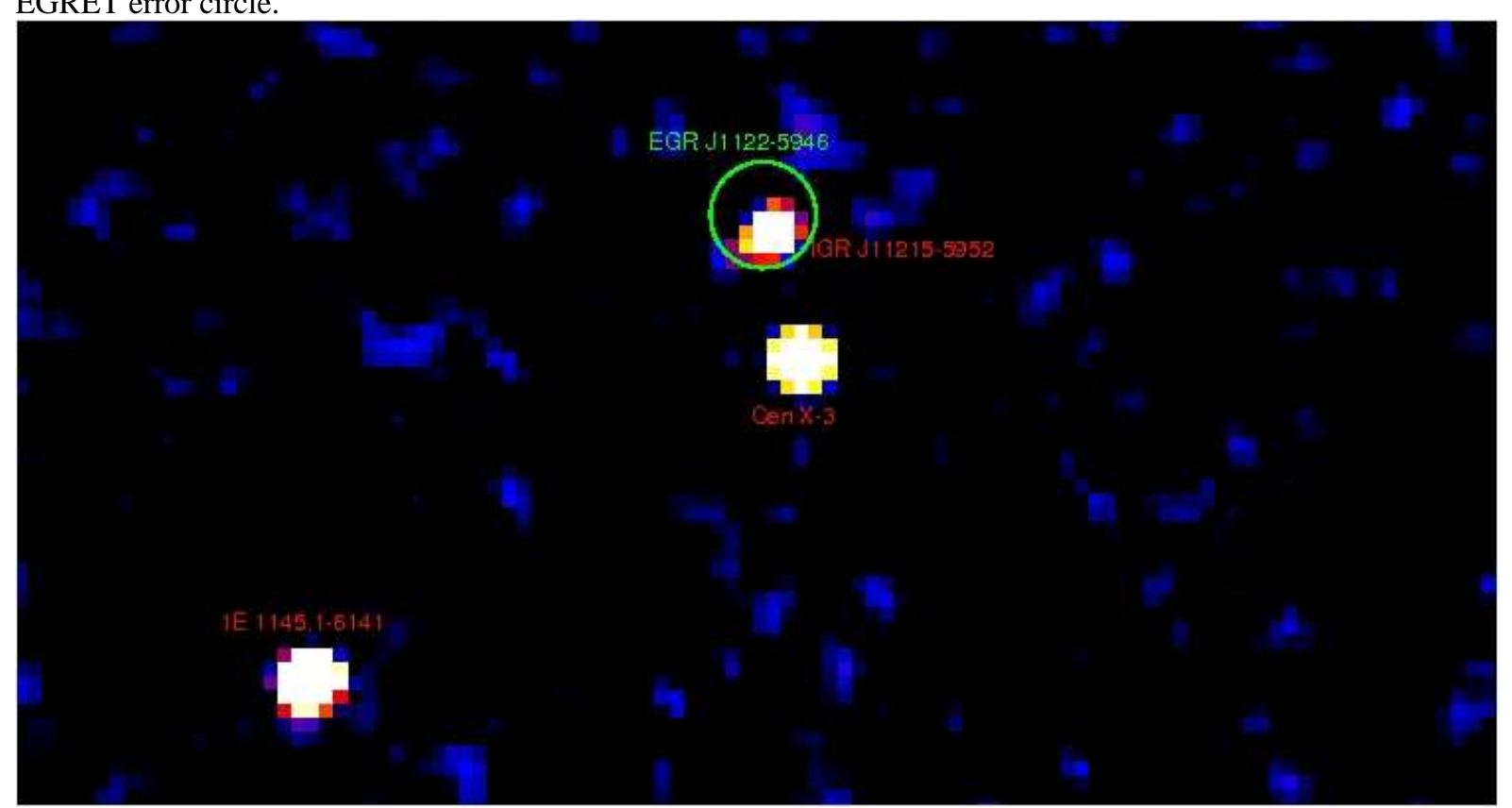

Figure 4: IBIS 20-100 keV significance mosaic image ( $\sim 5 \mathrm{ks})$ taking into account only the ScWs during which the SFXT IGR J11215-5952 was active. It is evident the spatial correlation between the SFXT and the unidentified source EGR J1122-5946 (green error circle). Contrary to Fig. 3, we note that the Be HMXB IGR J11435-6109 has not been detected because it was non active during such very short exposure time. 
3EG J1837-0423 and HESS J1841-055 (Sguera et al. 2008 submitted). Additional evidences for the existence of such a new class of galactic fast transient $\mathrm{MeV} / \mathrm{TeV}$ emitters is also provided by very recent AGILE and GLAST discoveries on the galactic plane of several unidentified transient sources lasting only a few days (Chen et al. 2007, Pittori et al. 2008, Longo et al. 2008, Cheung et al. 2008). We stress that flaring high energy emission could be common among HMXBs, this is supported by the fact that recently the supergiant HMXB Cygnus X-1 has been detected at TeV energies during a short flare lasting $\sim 80$ minutes (Albert et al. 2007). Interestingly, such $\mathrm{TeV}$ flare was simultaneous to a $20-40 \mathrm{keV}$ hard X-ray flare detected by IBIS at $\sim 1.5$ Crab level (Turler et al. 2006). Short TeV flares have been also detected in two other HMXBs, LS 5039 (De Naurois 2006, Paredes 2008) and LS I +61 303 (Paredes 2008).

As for the physical mechanism responsible for the fast gamma-ray flares, it is important to point out that four HMXBs and no LMXBS have been detected at $\mathrm{MeV} / \mathrm{TeV}$ energies, to date. This fact points to the need of having a bright and massive OB star as the source of seed photons (for the Inverse Compton emission) and target nuclei (for hadronic interactions). Sguera et al. (2008 submitted) proposed a theoretical mechanism able to explain the flaring $\mathrm{MeV}$ emission from the SFXT AX J1841.0-0536. In this scenario, AX J1841.0-0536 is a low magnetized pulsar which, due to accretion of a massive clump of material from the supergiant companion, undergoes sporadic changes to a transient Atoll-state where a magnetic tower can produce transient jets and as a consequence high energy transient emission. After the collision with the massive clump, everything comes back to the normal state. Moreover, Walter (2007b) suggested that HMXBs accreting dense clumps of material from the stellar wind could be transient $\mathrm{MeV} / \mathrm{TeV}$ sources if the column density is large enough i.e. $\geq \sim 10^{23} \mathrm{~cm}^{-2}$. Since such column densities have been observed in specific SFXTs during fast X-ray flares, the detection of fast $\mathrm{MeV} / \mathrm{TeV}$ flares on a few hours timescale it is not unexpected. Specifically, protons trapped in the outer and closed regions of a neutron star magnetosphere could be accelerated up to a Lorentz factor of $\sim 10^{8}$ by multiple scattering of Alfvén waves in or close to the accretion column and then interact with dense clump of materials at the magnetospheric radius producing $\gamma$-rays through inelastic $p p$ collisions and the subsequent decays. The duration and strength of the $\gamma$-rays flares should depend on the size of the clumps, structure of the wind and magnetic strength.

In summary, our results show that hard fast X-ray transients could represent a new class of galactic $\mathrm{MeV} / \mathrm{TeV}$ emitters. The high energy flaring emission from the binary system is expected only for a very small fraction of time so that fast $\mathrm{MeV} / \mathrm{TeV}$ flares are not easy to detect. Further multiwavelength observations and deeper studies in radio, X-rays (i.e. XMM, Chandra and Swift/XRT), soft gamma-rays (i.e. INTEGRAL), $\mathrm{MeV}$ and $\mathrm{GeV}$ frequencies (i.e. AGILE and GLAST) are strongly needed in order to support or reject our proposed scenario.

\section{References}

[1] Abdo, A. A., Allen, B., Berley,D., et al. 2007, ApJ, 658L, 33

[2] Aharonian F., Akhperjanian, A.G., Aye, K.M., et al. 2005a, Science, 309, 746

[3] Aharonian F., Akhperjanian, A.G., Aye, K.M., et al. 2005b, A\&A, 442, 1

[4] Albert, J., et al. 2006, Science, 312, 1771 
[5] Albert, J., Anderhub, H., Antoranz, P., et al. 2007, ApJ, 665, L51

[6] Bird, A. J., Malizia, A., Bazzano, A. et al. 2007, ApJS, 170, 175

[7] Bosch-Ramon et al. 2008, iastro-ph Modern Physics D, proceedings of HEPRO meeting, held in Dublin, in September 2007

[8] Casandjian, J-M \& Grenier, I.A. 2008, A\&A, in press,(arXiv:0806.0113),

[9] Chen, A., Vercellone, S., Giuliani, A., et al. 2007, ATEL 1308

[10] Cheung et al. 2008, ATEL 1788

[11] De naurois M., talk presented at the conference: the TeV to KeV connection, Rome, October 2006

[12] Halpern, J. P.; Eracleous, M.; Mukherjee, R., et al. 2001, ApJ, 551, 1016

[13] Halpern, J. P.; Camilo, F.; Giuliani, A., et al. 2008,'astro-ph 00810.0008; to appear in ApJ Letters

[14] Hartman, R. C., Bertsch, D. L., Bloom, S. D., et al. 1999, ApJS, 123, 79

[15] Longo, F., Chen, A., Vercellone, S., et al. 2008, ATEL 1492

[16] Negueruela, I., Smith, D. M., Reig, P., et al. 2006, ESA SP-604, 1, 165

[17] Negueruela, I., Torrejón, J.M., Reig, P. et al. 2008, AIP Conference Proceedings, 1010, 252

[18] Paredes J.M., 2008, 'astro-ph 0010.4428

[19] Pittori, C., Verrecchia, F., Santolamazza, P., et al. 2008, ATEL 1394

[20] Reynolds A.P., Parmar, A.N.; Hakala P.J., et al. 1999,A\&AS,134,287

[21] Ribo 2008, 2008arXiv0801.2906, ChJAA, in press, Frascati Workshop 2007, Vulcano, Italy

[22] Romano, P.; Sidoli, L.; Mangano, V.; et al. 2007, A\&A, 469L, 5

[23] Sguera, V., Romero, G.E., Bazzano, A., et al. 2008, submitted to ApJ

[24] Sguera, V., Bazzano, A., Bird, A. J., et al. 2006, ApJ, 646, 452

[25] Sguera, V., Malizia A., Bazzano, A., et al. 2006b, ATEL 1313

[26] Sguera, V., Barlow, E. J., Bird, A. J., et al. 2005, A\&A, 444, 221

[27] Sidoli, L.; Paizis, A.; Mereghetti, S., 2006, A\&A, 450L, 9

[28] Sidoli, L.; Romano, P.; Mereghetti, S. et al. 2007, A\&A, 476, 1307

[29] Ubertini, P., Lebrun, F., Di Cocco, G., et al. 2003, A\&A, 411, L131

[30] Turler et al. 2006, ATEL 911

[31] Walter, R. \& Zurita Heras, J. 2007, A\&A, 476, 335

[32] Walter, R., 2007b, Astrophysics and Space Science, 309, 5

[33] Winkler, C.; Courvoisier, T. J.-L.; Di Cocco, G.; 2003, A\&A, 411, L1

[34] Wood K.S., Meekins J.F.; Yentis D.J., et al. 1984, ApJS, 56, 507 